\title{
ESTRUTURA DE GOVERNANÇA E VALOR DAS COMPANHIAS ABERTAS BRASILEIRAS
}

\section{RESUMO}

A governança corporativa visa a aumentar a probabilidade de os fornecedores de recursos garantirem para si o retorno sobre seu investimento, por meio de um conjunto de mecanismos no qual se inclui o Conselho de Administração. Este artigo investiga se a estrutura de governança foi relevante para o valor das companhias abertas no Brasil entre 1998 e 2000. Três variáveis de governança foram consideradas: separação dos cargos de diretor executivo e presidente do conselho (DE), tamanho do conselho (TOT) e grau de independência do conselho (INDEP). Utilizou-se uma regressão múltipla do tipo seção transversal. DE foi a variável com os resultados mais importantes, apresentando evidência de que, em média, as empresas que têm pessoas distintas ocupando os cargos de diretor executivo e presidente do conselho são mais valorizadas pelo mercado.

\section{Alexandre Di Miceli da Silveira \\ FEA/USP}

Lucas Ayres B. de C. Barros

FEA/USP

\section{Rubens Famá}

FEA/USP

\begin{abstract}
Corporate governance seeks to increase the probability that suppliers of financial resources will assure themselves the return on their investment through a set of mechanisms including the Board of Directors. This paper investigates if the governance structure was important to the value of Brazilian listed firms from 1998 to 2000. Three governance variables were considered: the separation of chief executive officer and chairman positions (DE), board size (TOT), and board independence (Indep). The multiple regression method with cross sectional data was applied. The variable chief executive officer as chairman (DE) showed the most important results, with evidence that, on average, firms with different people in charge of the chief executive officer and chairman positions have higher market value.
\end{abstract}

PALAVRAS-CHAVE Governança corporativa, conselho de administração, valor da empresa, teste empírico, problema de agência. KEY WORDS Corporate governance, board of directors, firm value, empirical test, agency problem. 


\section{INTRODUÇÃo}

A presença de algum grau de separação entre propriedade e gestão na maioria das grandes corporações faz com que os executivos não carreguem todo o ônus financeiro de suas decisões. Nesse contexto, Jensen e Meckling (1976, p. 308) descrevem a existência de uma relação de agência entre acionistas e administradores, na qual os últimos atuam como agentes dos primeiros, sendo pagos para agir sempre de acordo com o interesse dos proprietários da corporação. Muitas vezes, entretanto, os gestores tomam decisões tendo em vista a maximização de sua utilidade pessoal e não o melhor interesse dos acionistas, resultando em expropriação da riqueza dos mesmos. A minimização dos prejuízos causados por esse problema, denominado por Jensen e Meckling (1976, p. 308) de problema de agência dos gestores, depende da presença de um conjunto de mecanismos internos e externos para harmonizar a relação entre as partes. A esse conjunto de mecanismos de incentivo e controle para minimização dos problemas de agência dá-se o nome de governança corporativa ${ }^{1}$. A relação entre governança corporativa e o problema de agência dos gestores pode ser visualizada na Figura 1.

Conforme observado na Figura 1, o Conselho de Administração é um dos principais mecanismos para alinhamento dos interesses de acionistas e gestores no sistema de governança corporativa de uma companhia. Além do Conselho de Administração, mecanismos internos - como o sistema de remuneração e a posse de ações por parte dos executivos - e mecanismos externos - como a obrigatoriedade da divulgação de informações periódicas sobre a companhia, a presença de um mercado de aquisição hostil e a existência de um mercado de trabalho competitivo - também são elementos importantes para a redução dos problemas de agência resultantes de tomadas de decisão que não tenham maximizado a riqueza dos acionistas por parte dos executivos.

Proliferaram, nos últimos anos, documentos conhecidos como "Códigos das Melhores Práticas de Governança Corporativa" (ver Anexo A), elaborados por reguladores e participantes dos mercados financeiros em diversos países, incluindo o Brasil. Dentre as práticas de governança mais recomendadas nesses documentos, destaca-se a necessidade de uma participação ativa e independente do Conselho de Administração, que pode ser alcançada por meio de uma estrutura de governança com:

- pessoas distintas ocupando os cargos de diretor executivo e presidente do conselho;
- maior participação possível de membros independentes no conselho;

- um conselho com número adequado de membros.

Praticamente todos os códigos de governança ressaltam a importância de um Conselho de Administração composto por uma maioria de membros externos - não executivos - na companhia. Essa recomendação reflete a idéia intuitiva de que a principal função do conselho é monitorar a gestão da empresa e que somente conselheiros externos profissionais podem ser monitores eficazes. Segundo os códigos de governança, um Conselho de Administração dominado por executivos pode atuar como um mecanismo de defesa dos gestores. Essa intuição é corroborada pelos argumentos de Jensen (2001, p. 52), segundo o qual os conselheiros internos - executivos da companhia - têm menor probabilidade de monitorar o desempenho do diretor executivo, haja vista que a evolução de suas carreiras depende em boa parte do próprio diretor executivo. Segundo Jensen (2001, p. 52), "como a possibilidade de uma atitude de animosidade e revide do diretor executivo é muito grande, é quase impossível para aqueles que se reportam diretamente a ele participarem de forma aberta e crítica na avaliação e monitoramento de tal diretor. Portanto, o único membro interno (executivo) do Conselho de Administração deve ser o diretor executivo".

Diversas pesquisas procuram relacionar a composição do Conselho de Administração com o desempenho da empresa. Nesses trabalhos, a pergunta geral a ser respondida é: "será que um conselho composto por uma maioria de membros externos - não executivos melhora o desempenho da empresa?". Um método utilizado tem sido o de examinar a correlação entre as medidas contábeis de desempenho e a proporção de conselheiros externos no conselho. Baysinger e Butler (1985) e Bhagat e Black (2002) realizaram testes com tal metodologia, não encontrando relação significativa entre medidas contábeis de desempenho e proporção de membros externos no conselho. Hermalin e Weisbach (1991) e Bhagat e Black (2002), adotando o $\mathrm{Q}$ de Tobin como medida de desempenho, utilizaram análise de correlação, não encontrando, entretanto, relação estatística significativa entre esse indicador e a composição do conselho. Bhagat e Black (1999), por sua vez, encontraram relação significativa entre composição do conselho e desempenho corporativo, constatando que os conselhos de empresas norte-americanas com maioria de membros independentes compor- 
tam-se de forma diferente dos conselhos sem essa maioria. Algumas diferenças parecem contribuir para o aumento do valor da empresa, enquanto outras parecem contribuir para sua diminuição.

Entretanto, ao contrário do senso comum proposto pelos diversos códigos de governança, Bhagat e Black (1999, p. 948) afirmam que não existe uma evidência convincente de que uma maior independência do conselho, representada por uma maioria de conselheiros não-executivos da companhia, seja correlacionada com maior lucratividade da empresa ou crescimento mais rápido. Segundo os autores, não existe apoio empírico para a proposição corrente de que as empresas devam ter uma "supermaioria de independentes no conselho" - conselhos com apenas um ou dois executivos internos, conforme definição dos autores. Pelo contrário, os autores afirmam que existem evidências de que empresas com supermaioria independente no conselho são menos lucrativas do que outras, já que apresentaram pior desempenho quando comparadas com outras empresas do estudo. Como conclusão, os autores sugerem que é interessante incluir um número moderado de internos - executivos - no Conselho de Administração.

Os resultados do estudo de Bhagat e Black (1999) são corroborados pelo estudo de Yermack (1996), que relata uma correlação negativa significativa entre a proporção de conselheiros independentes e o Q de Tobin, por meio de uma regressão múltipla. O autor não encontra, entretanto, correlação significativa entre composição do conselho e várias outras medidas de desempenho. Utilizando uma técnica de equações simultâneas, Barnhart e Rosenstein (1998) encontraram uma relação curvilínea, com um coeficiente negativo significativo, entre o Q de Tobin e a proporção de membros independentes no conselho, afirmando que as empresas com conselhos altamente independentes possuem valor menor. Os resultados da relação entre composição do conselho, desempenho corporativo e valor da empresa são difusos, com a maioria dos estudos encontrando pouca correlação. Entretanto, é interessante notar que alguns estudos recentes relatam a evidência de uma relação negativa significativa entre uma alta proporção de membros independentes no conselho e o desempenho da empresa, exatamente o oposto da sabedoria convencional.

Com relação ao tamanho do Conselho de Administração, alguns estudiosos acreditam que a efetividade do conselho diminui na medida em que ele ultrapassa um certo número de membros. Jensen (1993, p. 867) argumenta que um conselho "superpovoado" possui menor probabilidade de funcionar de forma efetiva e maior probabilidade de ser controlado pelo diretor executivo. Seu argumento é consistente com a teoria de comportamento organizacional, que indica um declínio da produtividade à medida que os grupos de trabalho aumentam de tamanho. Segundo Jensen (2001, p. 52), "os conselhos com mais de sete ou oito membros possuem uma probabilidade menor de funcionar de forma eficaz, tornando-se mais fáceis de serem controlados pelo diretor executivo".

Algumas pesquisas procuram verificar a relação entre o tamanho do Conselho de Administração e o desempenho e o valor da empresa. Utilizando o $Q$ de Tobin como estimativa do valor da empresa, Yermack (1996) encontra uma relação inversa entre o tamanho do conselho e o valor da empresa, isto é, conselhos maiores relacionados com menor valor das companhias. Segundo o autor, "o resultado é robusto para muitos controles, como tamanho da empresa, setor de atuação, posse de ações por parte dos executivos, oportunidades de crescimento e estruturas alternativas de governança. Empresas com menores conselhos também apresentam melhores índices financeiros e maiores incentivos ao bom desempenho do diretor executivo, por meio de compensação e ameaça de demissão". Eisenberg, Sundgren e Wells (1998), de forma similar, encontram uma correlação negativa entre o tamanho do conselho e os indicadores de rentabilidade para 900 pequenas e médias empresas finlandesas. Füerst e Kang (2000) encontram evidências de que o aumento do tamanho do Conselho de Administração possui um impacto negativo no desempenho da empresa. Na mesma linha de pesquisa, Brown e Maloney (1998, p. 25) afirmam que, em situações de aquisição hostil, conselhos menores predizem maiores retornos do preço das ações para as empresas compradoras. Entretanto, Dalton e Daily (2000), utilizando uma técnica de meta-análise, chegam a um resultado oposto ao dos autores anteriores, concluindo que maiores conselhos são associados com melhor desempenho financeiro, independentemente de como esse desempenho é medido.

O senso comum sugere que empresas com uma estrutura de governança mais adequada às práticas recomendadas pelos "Códigos das Melhores Práticas de Governança Corporativa" devem ser mais bem avaliadas pelo mercado no preço de suas ações do que empresas com uma estrutura de governança menos adequada, ceteris paribus. Parece estar implícita, assim, a hipótese de que a estrutura de governança corporativa da empresa afeta seu valor de mercado. Nesse contexto, o 
objetivo do presente estudo é contribuir para a discussão do tema, verificando a existência de relações estatisticamente significativas entre variáveis de governança corporativa e valor das empresas.

\section{DEFINIÇÃO OPERACIONAL DAS VARIÁVEIS}

O estudo investiga se a estrutura de governança corporativa da companhia aberta brasileira relaciona-se significativamente com seu valor de mercado. A estrutura de governança atua como variável independente, enquanto o valor da empresa atua como variável dependente. São utilizadas variáveis de controle selecionadas a fim de isolar sua influência sobre o relacionamento entre as variáveis de interesse do estudo. A escolha das variáveis - independentes, dependentes e de controle - tem por base os estudos de Bhagat e Black (2002), Füerst e Kang (2000), Barnhart e Rosenstein (1998) e Yermack (1996), que analisam a relação entre estrutura do conselho de administração e desempenho e valor corporativo.

\section{Variáveis independentes de governança}

A estrutura de governança, nesta pesquisa, é definida unicamente pelas características de seu Conselho de Administração, com a utilização de três variáveis independentes:

- Presença de diretor executivo ocupando o cargo de presidente do conselho da empresa - é representada por uma variável binária, onde:

$\mathrm{DE}=1$, se os cargos de diretor executivo e presidente do conselho forem ocupados pela mesma pessoa, isto é, se os cargos forem unificados;

$\mathrm{DE}=0$, se os cargos forem ocupados por pessoas diferentes.

- Grau de independência do Conselho de Administração - é a diferença entre a proporção de membros não executivos (externos) e membros executivos (internos) no conselho, dada pela seguinte expressão:

$I N D E P=\left(\frac{E X T}{T O T}\right)-\left(\frac{I N T}{T O T}\right)$

Onde:

INDEP - grau de independência do Conselho de Administração;

TOT - número total de membros do conselho;

EXT - número de membros não executivos da em- presa (externos) no conselho;

INT - número de membros executivos da empresa (internos) no conselho.

- Tamanho do conselho - é dado pelo número total de membros do órgão (TOT).

Além de verificar se a estrutura de governança possui relação com o valor das companhias abertas brasileiras, o estudo também verifica a hipótese de que as empresas com estrutura de governança mais adequada às recomendações do "Código das Melhores Práticas de Governança Corporativa" do Instituto Brasileiro de Governança Corporativa (IBGC) e da "Cartilha de Governança Corporativa" da Comissão de Valores Mobiliários (CVM) apresentaram maior valor de mercado no período analisado. Esses códigos de governança sugerem que as empresas devam ter:

- pessoas distintas ocupando os cargos de diretor executivo e presidente do Conselho de Administração;

- maior proporção possível de membros não executivos no Conselho de Administração;

- tamanho do Conselho de Administração entre cinco e nove membros.

A adoção do Código do IBGC e da Cartilha de Governança da CVM justifica-se por serem eles os principais documentos sobre o tema no Brasil, tendo sido elaborados a partir dos principais códigos de governança internacionais.

\section{Variáveis dependentes de valor}

São utilizados dois indicadores de valor a fim de testar a consistência dos resultados:

- Q de Tobin - esse indicador tem sido utilizado em vários estudos com testes empíricos sobre governança corporativa, como os de Barnhart e Rosenstein (1998), Bhagat e Black (2002) e Yermack (1996). Tobin e Brainard (1968) e Tobin (1969) definiramno como:

Q de Tobin $=\frac{\text { Valor de Mercado das Ações + Valor de Mercado das Dívidas }}{\text { Valor de Reposição dos Ativos }}$

O cálculo do $\mathrm{Q}$ de Tobin na prática é bastante difícil. Com exceção do valor de mercado das ações, diretamente observável pela cotação na bolsa de valores, os outros componentes da fórmula, o valor de mercado das dívidas e o valor de reposição dos ativos da empresa, não são observáveis diretamente, em geral. $\mathrm{Na}$ ausência da disponibilidade de medidas diretas, 
apenas medidas indiretas podem ser estimadas. No estudo, o Q de Tobin é estimado pela aproximação simplificada proposta por Chung e Pruitt (1994, p. $72)^{2}$, que o definem como:

$$
\mathrm{Q} \text { de Tobin }=\frac{V M A O+V M A P+D I V T}{A T}
$$

Onde:

VMAO - valor de mercado das ações ordinárias; VMAP - valor de mercado das ações preferenciais; DIVT - valor contábil das dívidas de curto e longo prazo menos o ativo circulante, após a exclusão do valor dos estoques;

AT - ativo total da companhia.

- Valor da Empresa sobre Ativo Total (FIRMVSAT) esse indicador é uma alternativa ao Q de Tobin, sendo calculado pelo quociente:

FIRMVSAT $=\frac{\text { FirmValue }}{A T}$

Onde:

Firm Value - indicador calculado pelo sistema de informações Economática ${ }^{\circledR}$ por meio da expressão:

Firm Value $=$ Cotação* Total de Ações + Debêntures de curto e longo prazos + Financiamentos de curto e longo prazos + Adiantamentos de Contrato de Câmbio - Disponibilidades e Investimentos de Curto Prazo;

AT - ativo total da empresa.

\section{Variáveis de controle}

As variáveis de controle foram selecionadas com base na possivel influência que exercem sobre as variáveis dependentes de valor (Q e FIRMVSAT) e independentes de governança (INDEP, TOT e DE). Caso as variáveis de controle, que são variáveis independentes, possuam forte correlação com algumas dessas variáveis e sejam omitidas no modelo, a relação entre as variáveis de interesse pode não ser evidenciada de forma correta. Assim, as seguintes variáveis de controle foram utilizadas:

- Setor de atuação - foi adotado o critério de classificação do Standard Industrial Code (SIC) norte-americano do tipo Division;

- Índice de liquidez da ação, calculado pelo sistema de informações Economática ${ }^{\circledR}$ para períodos de 12 e 24 meses, tendo por base o volume de negociações com o papel;

- Tamanho da empresa, medido por sua receita operacional ou por seu ativo total;

- Estrutura de financiamentos, dada pela razão entre as dívidas financeiras totais e o ativo total das empresas; e

- Variáveis de desempenho. A rentabilidade histórica das empresas foi mensurada a partir de diversas definições operacionais, incluindo o cálculo do lucro líquido médio dos quatro trimestres do ano escolhido para a análise dividido pelo ativo médio ou pelo patrimônio líquido médio correspondente. Alternativamente, utilizou-se o mesmo procedimento, porém com a substituição do lucro líquido pelo lucro operacional ou lucro operacional próprio ou, ainda, pelo lucro antes de juros, impostos, depreciação e amortização (EBITDA). A política de dividendos das empresas também foi levada em conta, representada pelo índice de payout médio dos cinco anos anteriores ao período analisado e pelo quociente entre o valor dos dividendos pagos e o ativo total da empresa dentro de cada ano estudado.

\section{MODELO EMPÍRICO}

Como forma de investigar a suposta existência de um relacionamento significativo entre as variáveis de governança corporativa e o valor das empresas abertas brasileiras e avaliar se as empresas que seguem as recomendações dos códigos do IBGC e da CVM são as que alcançam melhor avaliação de mercado, foi estimado o seguinte modelo empírico:

$$
\begin{aligned}
& V_{i}=\alpha+\beta_{1}{ }^{*} \operatorname{INDEP}_{i}+\beta_{2}{ }^{*} \log \left(\mathrm{TOT}_{\mathrm{i}}\right)+\beta_{3}{ }^{*} \log \left(\mathrm{TOT}_{\mathrm{i}}\right)^{2}+ \\
& \beta_{4}{ }^{*} \mathrm{DE} E_{i}+\sum_{j=1}^{k} \delta_{j}{ }^{*} V C_{j i}+\varepsilon_{i}
\end{aligned}
$$

Onde:

$\mathrm{V}_{i}$ - medidas de valor da i-ésima empresa: $\mathrm{Q}$ de Tobin ou FIRMVSAT;

INDEP $_{i}$ - grau de independência do Conselho de Administração da i-ésima empresa;

$\mathrm{TOT}_{i}$ - tamanho do Conselho de Administração da iésima empresa;

$\mathrm{DE}_{i}$ - presença de diretor executivo ocupando o cargo de presidente do conselho na i-ésima empresa;

$\mathrm{VC}_{\mathrm{ji}}$ - j-ésima variável de controle, de um total de $\mathrm{k}$ variáveis, medida para a i-ésima empresa;

$\varepsilon_{i}$ - termo de erro que capta o componente não sistemático, a parcela de $\mathrm{V}$ não explicada pelo modelo.

Partindo da hipótese de que quanto maior é o grau de independência do conselho (INDEP), maior é o va- 
lor da empresa (V), espera-se que o coeficiente estimado $\beta_{1}$ seja positivo e significativo estatisticamente. Com relação ao tamanho do conselho (TOT), a hipótese é de que existe uma faixa ótima para os conselhos, com cinco a nove membros. Dessa forma, para captar o comportamento proposto, inseriu-se um termo quadrático na regressão - TOT ao quadrado. Além disso, o uso dessa variável, que é eminentemente discreta, em escala logarítmica resultou em modelos com melhor qualidade estatística, razão pela qual apenas as especificações com log(TOT) são reportadas. Para que a hipótese da faixa ótima para o tamanho do conselho se confirme, espera-se que os coeficientes estimados $\beta_{2}$ e $\beta_{3}$ sejam significativos estatisticamente e tenham sinais positivos e negativos, respectivamente. Por fim, para que se corrobore a hipótese de que é saudável para a empresa a presença de pessoas distintas ocupando os cargos de diretor executivo e presidente do conselho, espera-se que o valor do coeficiente estimado $\beta_{4}$ seja significativo estatisticamente e negativo, apontando para uma relação inversa entre valor da empresa e unificação dos cargos de diretor executivo e presidente do conselho. Caso os valores dos coeficientes acima se mostrem estatisticamente significativos mas com sinal inverso ao proposto, será constatada a relevância da estrutura de governança, porém de forma oposta à recomendada pelos códigos do IBGC e da CVM.

A análise dos modelos foi feita com base nos critérios estatísticos usuais, incluindo a avaliação dos níveis de significância estatística dos regressores - valores de probabilidade das estatísticas t e F -, do R-quadrado ajustado e do Critério de Informação de Schwarz. O R-quadrado indica em que medida o modelo explica o comportamento da variável dependente. O Critério de Informação de Schwarz, por sua vez, auxilia na seleção de modelos ao mesmo tempo parcimoniosos no número de variáveis independentes e com bom ajuste e significância estatística.

A análise da adequação dos modelos estimados aos pressupostos de um Modelo de Regressão Linear (MRL) utilizou como principais instrumentos de diagnóstico o teste de heterocedasticidade de White e o teste de normalidade de resíduos de Jarque-Bera. O teste de White pode ser considerado um teste geral, já que verifica a adequação do modelo a três pressupostos de um MRL: a homocedasticidade dos termos de erro variância constante -, a especificação linear correta do modelo e a independência dos erros em relação aos regressores. O teste de Jarque-Bera verifica a adequação do modelo com relação à suposição de distribui- ção Normal dos termos de erro, outro pressuposto importante de um MRL. As observações extremas - outliers - e sua influência sobre as estimativas também foram analisadas seguindo critérios como a distância de Cook e a avaliação dos resíduos padronizados. Por fim, problemas de multicolinearidade entre as variáveis foram verificados com o teste do Número de Condição.

\section{POPULAÇ̃̃O, AMOSTRAGEM E COLETA DE DADOS}

As empresas componentes das amostras foram sorteadas aleatoriamente a partir do conjunto das companhias abertas não financeiras negociadas na Bovespa e que apresentaram, nos anos de 1998, 1999 e 2000, liquidez acima de um nível mínimo definido pelo pesquisador. A escolha de uma faixa de corte para a construção das amostras, ao invés da coleta de dados de todas as companhias abertas, deve-se à suposição de que as empresas com liquidez muito baixa possuem uma probabilidade menor de terem suas cotações adequadas a seu verdadeiro valor de mercado. Não obstante, o nível de liquidez mínimo, estipulado em 0,001\%3 do índice da empresa com maior liquidez, é essencialmente arbitrário, visto que não há qualquer padrão amplamente aceito na literatura para a determinação do grau de liquidez satisfatório em termos absolutos ou relativos. O valor de referência escolhido refletiu, então, a preocupação com o tamanho das amostras, de maneira a garantir a presença de um número suficiente de observações para a produção de inferências válidas. A fórmula utilizada para o cálculo da liquidez das ações é a adotada pelo sistema de informações Economática ${ }^{\circledR}$, de cujo banco de dados foi extraída parte dos dados da pesquisa.

Após a aplicação do critério de liquidez mínima, restaram 218 empresas nos anos de 1999 e 1998, e 215 empresas no ano de 2000, número significativo em relação ao total de companhias listadas na Bolsa de Valores de São Paulo ${ }^{4}$. Em face da conveniência estatística de uma amostragem do tipo aleatória, foram sorteadas 120 empresas para cada ano, cujos dados serviram de base para os testes. Foram utilizados dados secundários referentes ao valor de mercado das ações, variáveis de governança e demais variáveis de controle. Essas informações são disponibilizadas pelos sistemas de informações Economática ${ }^{\circledR}$, Bloomberg $^{\circledR}$ e Divulgação Externa ITR /DFP /IAN - Divext ${ }^{\circledR}-$ da Comissão de Valores Mobiliários. 


\section{RESULTADOS}

Os principais resultados obtidos com relação à significância estatística dos coeficientes na regressão múltipla entre as variáveis independentes de governança e as variáveis dependentes de valor estão resumidos na Tabela 1. O sinal entre parênteses representa o sinal do coeficiente estimado, enquanto o percentual representa o nível de significância estatística do coeficiente com base na estatística " $t$ " calculada.

Conforme os resultados da Tabela 1, a variável "diretor executivo como presidente do conselho" (DE) foi o indicador de governança com os resultados mais importantes do estudo, apresentando um coeficiente negativo e significante estatisticamente nos níveis de $1 \%$, $15 \%$ e $10 \%$ contra ambas as variáveis dependentes de valor Q de Tobin (Q) e valor da empresa sobre o ativo total (FIRMVSAT) nos anos de 2000, 1999 e 1998, respectivamente. Os resultados foram consistentes, em especial no ano de 2000, apresentando evidência de que, em média, as empresas que tiveram pessoas distintas ocupando os cargos de diretor executivo e presidente do conselho obtiveram maior valor de mercado, e corroborando a hipótese de que a adoção das recomendações do IBGC e da CVM para a separação dos cargos de diretor executivo e presidente do conselho é saudável para as empresas. O fato de que a relação entre DE e as variáveis dependentes mostrou-se substancialmente mais forte no ano de 2000 sugere que o mercado pode recentemente ter se tornado mais sensível às recomendações dos códigos de governança e recompensado mais as empresas que adotam a separação de cargos.

A variável tamanho do conselho (TOT) apresentou uma relação quadrática no nível de significância de 10\% contra a variável FIRMVSAT em 2000, e uma ausência de relação estatística significativa contra a variável Q. Com relação aos sinais dos coeficientes de TOT, era esperado que o sinal do coeficiente do termo linear

Figura 1 - 0 problema de agência dos gestores e a governança corporativa.

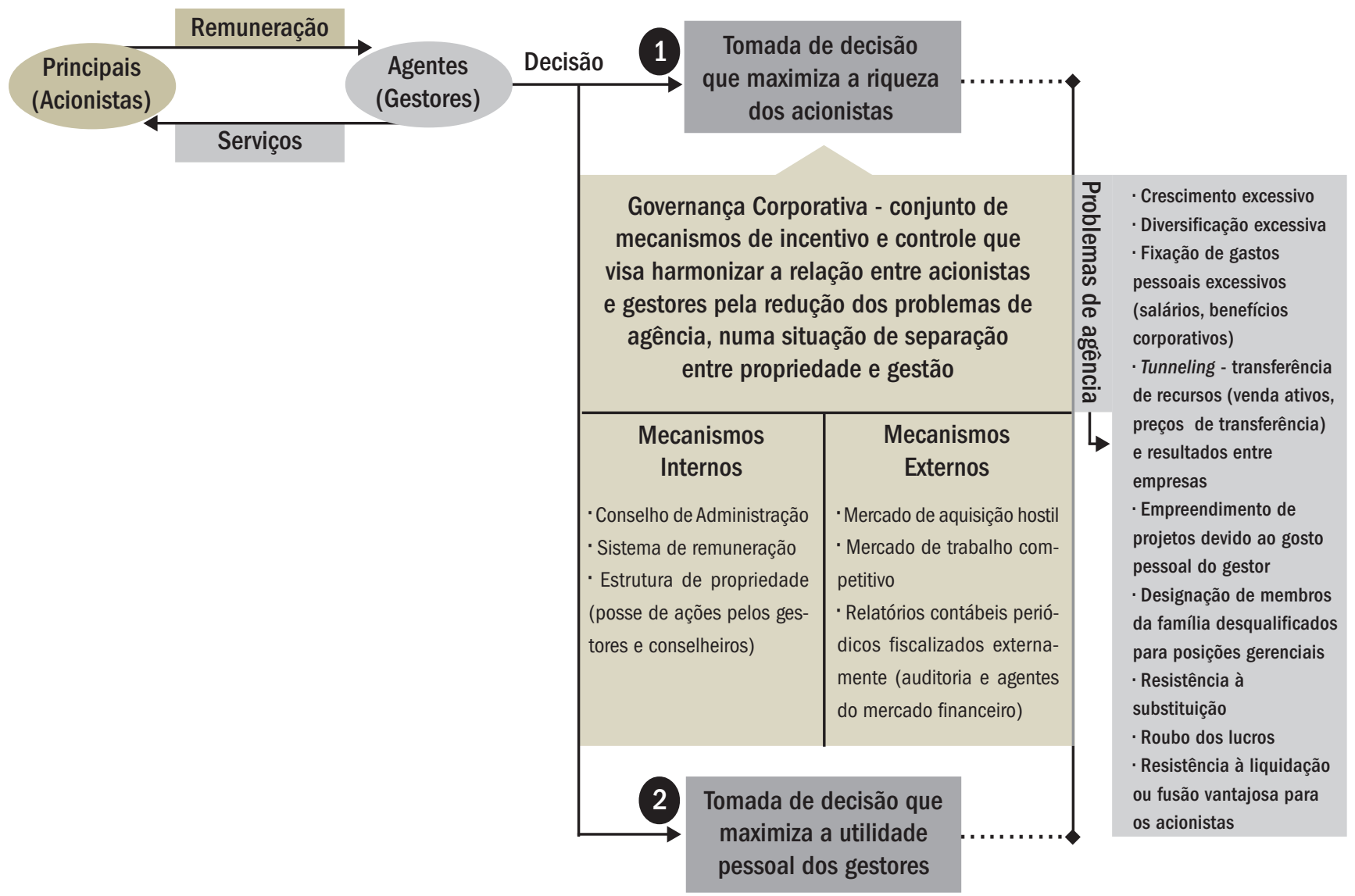


fosse positivo e que o sinal do coeficiente do termo quadrático fosse negativo, indicando uma concavidade para baixo da curva, de forma a corroborar as recomendações do IBGC e da CVM quanto à existência de uma faixa ótima de cinco a nove membros para o tamanho do conselho. E esse foi exatamente o resultado obtido no modelo que apresentou significância estatística, com os sinais dos coeficientes de TOT indican- do uma relação curvilínea negativa e um tamanho ótimo do conselho. Foi construído um gráfico - Figura 2 - para visualizar a relação não linear entre valor da empresa e número de membros do conselho. Nele, pode-se observar um tamanho de conselho ótimo entre cinco a seis membros, com o valor máximo de FIRMVSAT correspondendo a um conselho de 5,15 membros. Por fim, A variável INDEP apresentou signi-

Tabela 1 - Resumo dos resultados da pesquisa.

\begin{tabular}{|c|c|c|c|c|c|}
\hline & \multicolumn{5}{|c|}{ VARIÁVEIS DE GOVERNANÇA (INDEPENDENTES) } \\
\hline & & DE & TOT & $\mathrm{TOT}^{2}$ & INDEP \\
\hline \multirow{6}{*}{ 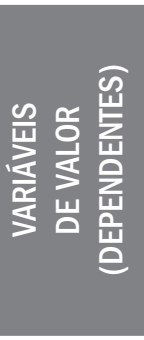 } & Q00 & (-) $1 \%$ & & & \\
\hline & FIRMVSAT00 & (-) $1 \%$ & (+) $10 \%$ & (-) $5 \%$ & \\
\hline & Q99 & (-) $15 \%$ & & & (+) $10 \%$ \\
\hline & FIRMVSAT99 & (-) $15 \%$ & & & \\
\hline & Q98 & (-) $10 \%$ & & & \\
\hline & FIRMVSAT98 & (-) $10 \%$ & & & \\
\hline \multirow{3}{*}{ 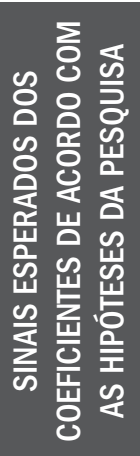 } & $\begin{array}{l}\mathrm{H}_{\mathrm{o}} \text { - irrelevância } \\
\text { da governança } \\
\text { corporativa }\end{array}$ & & & & \\
\hline & $\begin{array}{l}\mathrm{H}_{1} \text { - confirmação } \\
\text { das recomendações } \\
\text { do IBGC e CVM }\end{array}$ & - & + & - & + \\
\hline & $\begin{array}{l}\mathrm{H}_{2} \text { - sentido } \\
\text { oposto às } \\
\text { recomendações } \\
\text { do IBGC e CVM }\end{array}$ & + & - & + & - \\
\hline
\end{tabular}

Entre parênteses pode-se observar o sinal do coeficiente obtido no modelo, enquanto o percentual representa o nível de significância estatística do coeficiente com base na estatística $t$. Os sinais indicados para as hipóteses $\mathrm{H}_{0}, \mathrm{H}_{1}$ e $\mathrm{H}_{2}$ refletem os sinais esperados dos coeficientes de acordo com as hipóteses da pesquisa.

Tabela 2 - Relação entre a variável valor da empresa sobre ativo total (FIRMVSAT) e as variáveis de governança em 2000.

\begin{tabular}{|l|c|c|c|c|}
\hline \multicolumn{1}{|c|}{ VARIÁVEL } & COEFICIENTE & ERRO PADRÃO & ESTATÍSTICA T & PROB. \\
\hline Constante & 0,129728 & 0,192304 & 0,674599 & 0,5009 \\
\hline DE & $-0,125321$ & 0,031199 & $-4,016879$ & $\mathbf{0 , 0 0 0 1}$ \\
\hline INDEP & $-0,032955$ & 0,052086 & $-0,632709$ & 0,5279 \\
\hline LOG(TOT) & 0,381585 & 0,215447 & 1,771129 & $\mathbf{0 , 0 7 8 5}$ \\
\hline LOG(TOT)^2 & $-0,116358$ & 0,058388 & $-1,992859$ & $\mathbf{0 , 0 4 8 0}$ \\
\hline SERV & 0,097172 & 0,029009 & 3,349673 & 0,0010 \\
\hline DIVTSAT & 0,005077 & 0,000802 & 6,332191 & 0,0000 \\
\hline R-quadrado & 0,370514 & Critério de Schwarz & $-0,732829$ \\
\hline R-quadrado ajustado & 0,345989 & Prob. (estatística F) & 0,000000 \\
\hline
\end{tabular}


ficância estatística, de $10 \%$, apenas contra a variável de valor Q de Tobin em 1999, sugerindo que as empresas com maior INDEP - proporção de conselheiros externos no conselho - apresentaram, em média, maior $\mathrm{Q}$ de Tobin naquele ano.

É importante destacar que em alguns modelos os sinais dos coeficientes de uma ou mais variáveis de governança são opostos aos esperados. Em todos esses casos, porém, os coeficientes revelam-se insignificantes estatisticamente. Isso indica que os valores diferentes de zero observados são resultado de erro amostral com grande probabilidade e, portanto, não constituem evidência de que o relacionamento entre valor e estrutura de governança se dá no sentido contrário àquele suposto nas hipóteses desta pesquisa. Por outro lado, a insignificância dos coeficientes em diversas especificações sugere que a evidência em favor das hipóteses mencionadas é não conclusiva. De fato, apenas a variável DE apresentou resultados consistentes em todas as amostras. Possivelmente, as limitações metodológicas do estudo podem ser responsabilizadas por tais ambigüidades. É também possível supor, todavia, que os agentes dos mercados de capitais não atribuíram, por qualquer razão, importância significativa a aspectos específicos da governança corporativa, a exemplo do tamanho do Conselho de Administração e do grau de independência de seus membros, pelo menos após isolados os efeitos da variável "diretor executivo como presidente do conselho".
De acordo com os resultados apresentados na Tabela 8 , os testes de heterocedasticidade de White nas regressões entre as variáveis de valor Q e FIRMVSAT e as variáveis de governança apresentaram resultados aceitáveis - valor de probabilidade de 0,10 para 0 modelo apresentado na Tabela 3, e maior do que 0,25 para todos os outros modelos -, não rejeitando, nos níveis convencionais de significância, a hipótese nula de homocedasticidade dos resíduos para todos os modelos testados.

Os testes de normalidade dos resíduos de Jarque-Bera, apresentados também na Tabela 8 , apontam para a não rejeição, em todos os modelos, da hipótese nula, ou seja, de que os termos de erro se distribuem como uma Normal - valor mínimo de probabilidade de $0,21^{5}$. A utilização de duas variáveis intrinsecamente relacionadas TOT e TOT ao quadrado - resultou em Números de Condição (NC) altos, maiores do que 20, calculados para todos os modelos, o que indicaria problemas de multicolinearidade. A presença de multicolinearidade, entretanto, não afeta a significância das outras variáveis, conforme se comprova pela estimação de modelos sem a transformação quadrática de TOT, os quais apresentaram baixo NC e resultados de direção, magnitude e nível de significância das demais variáveis praticamente inalterados. Como a utilização de TOT ao quadrado é fundamental para testar a hipótese da faixa ótima para o tamanho do conselho e a multicolinearidade é causada apenas pelas variáveis TOT e TOT ao quadrado, não con-

Figura 2 - Relação entre valor (FIRMVSAT) e número de membros do conselho (TOT) com base no modelo da Tabela 2.

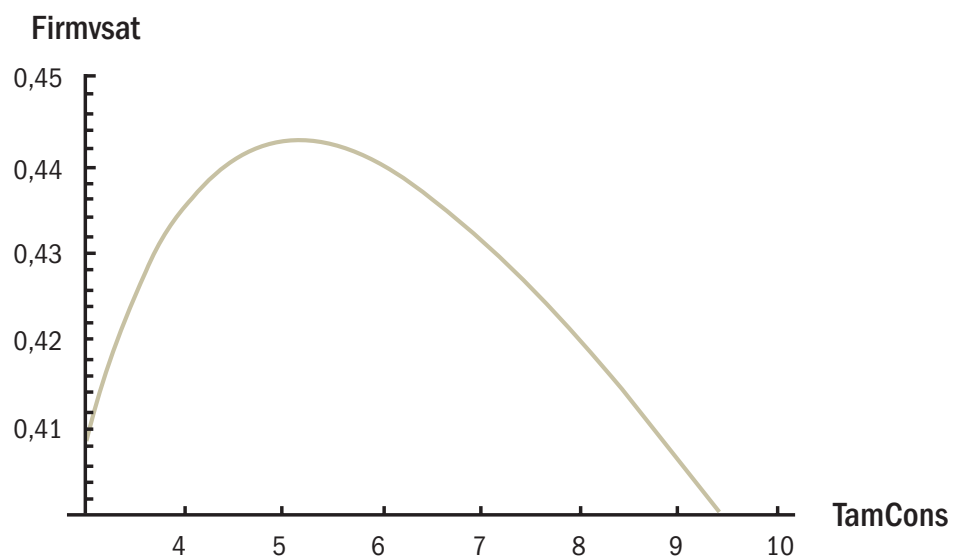

Tamanho ótimo para o conselho: 5,15 membros.

No eixo horizontal do gráfico, figuram os valores originais de TOT e não sua transformação logarítmica.

$58 \cdot \mathrm{ORAE} \cdot \mathrm{VOL} 43 \cdot \mathrm{N}^{\circ} 3$ 
taminando os resultados dos outros coeficientes, a questão da multicolinearidade não foi considerada um problema para os testes.

\section{CONSIDERAÇÕES FINAIS}

O objetivo principal do estudo foi verificar, estatisticamente, a existência de uma relação significativa entre governança corporativa e valor das companhias abertas brasileiras. Três variáveis de governança foram testadas contra as variáveis de valor em regressões lineares múltiplas do tipo seção transversal. São elas:

- a presença de pessoas distintas ocupando os cargos de diretor executivo e presidente do Conselho de Administração (DE);
- o tamanho - número de membros - do Conselho de Administração (TOT);

- o grau de independência do Conselho de Administração (INDEP).

A variável "diretor executivo como presidente do conselho" (DE) foi o indicador de governança com os resultados mais importantes do estudo, apresentando um coeficiente negativo e significante estatisticamente nos níveis de 1\%, 15\% e 10\% contra ambas as variáveis dependentes de valor $\mathrm{Q}$ de Tobin e valor da empresa sobre o ativo total (FIRMVSAT) nos anos de 2000, 1999 e 1998, respectivamente. Os resultados foram consistentes, apresentando evidência de que, em média, as empresas que tiveram pessoas distintas ocupando os cargos de diretor executivo e presidente do conselho obti-

Tabela 3 - Relação entre a variável Q de Tobin (Q) e as variáveis de governança em 2000.

\begin{tabular}{|l|c|c|c|c|}
\hline \multicolumn{1}{|c|}{ VARIÁVEL } & COEFICIENTE & ERRO PADRÃO & ESTATÍSTICA T & PROB. \\
\hline Constante & 0,715994 & 0,245812 & 2,912771 & 0,0041 \\
\hline DE & $-0,163405$ & 0,039794 & $-4,106228$ & $\mathbf{0 , 0 0 0 1}$ \\
\hline INDEP & $-0,011203$ & 0,067749 & $-0,165362$ & 0,8689 \\
\hline LOG (TOT) & $-0,118685$ & 0,276261 & $-0,429611$ & 0,6681 \\
\hline LOG (TOT)^2 & $-0,007985$ & 0,075279 & $-0,106074$ & 0,9157 \\
\hline DIVTSAT & 0,008226 & 0,000834 & 9,868334 & 0,0000 \\
\hline R-quadrado & 0,463767 & Critério de Schwarz & $-0,209281$ \\
\hline R-quadrado ajustado & 0,447010 & Prob. (estatística F) & 0,000000 \\
\hline
\end{tabular}

Tabela 4 - Relação entre a variável Q de Tobin (Q) e as variáveis de governança em 1999.

\begin{tabular}{|l|c|c|c|c|}
\hline \multicolumn{1}{|c|}{ VARIÁVEL } & COEFICIENTE & ERRO PADRÃO & ESTATÍSTICA T & PROB. \\
\hline Constante & 0.261386 & 0.244459 & 1.069241 & 0.2865 \\
\hline DE & -0.051573 & 0.037351 & -1.380777 & $\mathbf{0 . 1 6 9 2}$ \\
\hline INDEP & 0.121457 & 0.071674 & 1.694569 & $\mathbf{0 . 0 9 2 0}$ \\
\hline LOG (TOT) & 0.023583 & 0.270400 & 0.087215 & 0.9306 \\
\hline LOG (TOT)^2 & -0.020165 & 0.073429 & -0.274619 & 0.7839 \\
\hline DIVTSAT & 0.008051 & 0.000803 & 10.02144 & 0.0000 \\
\hline SERV & 0.186988 & 0.034527 & 5.415629 & 0.0000 \\
\hline R-quadrado & 0.438809 & Critério de Schwarz & 0.553410 \\
\hline R-quadrado ajustado & 0.419002 & Prob. (estatística F) & 0.000000 \\
\hline
\end{tabular}


veram maior valor de mercado, e corroborando a hipótese de que a adoção das recomendações do IBGC e CVM para separação dos cargos de diretor executivo e presidente do conselho é saudável para as empresas.

A variável tamanho do conselho (TOT) apresentou uma relação quadrática no nível de significância de 10\% contra a variável FIRMVSAT em 2000, e uma ausência de relação estatística significativa contra a variável $Q$. Era esperada, a partir das recomendações do IBGC e da CVM, a existência de uma faixa ótima para o tamanho do conselho. Na regressão, isso seria refletido em um comportamento não linear da variável TOT, com sinal do coeficiente do termo linear positivo e com o sinal do coeficiente do termo quadrático negativo, indicando uma concavidade para baixo da curva. Esse foi o resultado obtido na relação entre TOT e a variá- vel de valor FIRMVSAT em 2000, indicando que as empresas com um número intermediário de conselheiros - cinco a seis membros - obtiveram, em média, maior valor, corroborando as recomendações do IBGC e da CVM. A relação curvilínea negativa encontrada na relação entre TOT e FIRMVSAT em 2000 é similar aos resultados obtidos por Barnhart e Rosenstein (1998) na relação entre o $Q$ de Tobin e a proporção de membros independentes no conselho, utilizando uma técnica de equações simultâneas. O resultado geral, entretanto, de baixa correlação entre o tamanho do conselho e o valor corporativo, diverge dos resultados descritos por Hermalin e Weisbach (1991, p. 3), que afirmam que a maior parte da literatura empírica sobre conselhos de administração aponta para uma relação linear negativa significativa entre tamanho do conse-

Tabela 5 - Relação entre a variável valor da empresa sobre ativo total (FIRMVSAT) e as variáveis de governança em 1999.

\begin{tabular}{|l|c|c|c|c|}
\hline \multicolumn{1}{|c|}{ VARIÁVEL } & COEFICIENTE & ERRO PADRÃO & ESTATÍSTICA T & PROB. \\
\hline Constante & 0,542852 & 0,180029 & 3,015361 & 0,0030 \\
\hline DE & $-0,041194$ & 0,028366 & $-1,452241$ & $\mathbf{0 , 1 4 8 3}$ \\
\hline INDEP & 0,018996 & 0,054762 & 0,346880 & 0,7291 \\
\hline LOG (TOT) & $-0,283902$ & 0,201263 & $-1,410605$ & 0,1602 \\
\hline LOG (TOT)^2 & 0,059243 & 0,055152 & 1,074178 & 0,2843 \\
\hline DIVTSAT & 0,007594 & 0,000556 & 13,66491 & 0,0000 \\
\hline SERV & 0,146527 & 0,026866 & 5,453991 & 0,0000 \\
\hline R-quadrado & 0,575376 & Critério de Schwarz & $-0,796452$ \\
\hline R-quadrado ajustado & 0,560028 & Prob. (estatística F) & 0,000000 \\
\hline
\end{tabular}

Tabela 6 - Relação entre a variável Q de Tobin (Q) e as variáveis de governança em 1998.

\begin{tabular}{|l|c|c|c|c|}
\hline \multicolumn{1}{|c|}{ VARIÁVEL } & COEFICIENTE & ERRO PADRÃO & ESTATÍSTICA T & PROB. \\
\hline Constante & 0,442377 & 0,196703 & 2,248963 & 0,0261 \\
\hline DE & $-0,053944$ & 0,030139 & $-1,789863$ & $\mathbf{0 , 0 7 5 6}$ \\
\hline INDEP & $-0,004570$ & 0,059435 & $-0,076882$ & 0,9388 \\
\hline LOG (TOT) & $-0,224941$ & 0,224690 & $-1,001119$ & 0,3185 \\
\hline LOG (TOT)^2 & 0,062654 & 0,063153 & 0,992097 & 0,3228 \\
\hline SERV & 0,188560 & 0,026555 & 7,100654 & 0,0000 \\
\hline DIVTSAT & 0,006661 & 0,000675 & 9,863646 & 0,0000 \\
\hline R-quadrado & 0,501125 & Critério de Schwarz & $-0,980605$ \\
\hline R-quadrado ajustado & 0,480046 & Prob. (estatística F) & 0,000000 \\
\hline
\end{tabular}


lho e variáveis de desempenho corporativo. Foram realizadas regressões alternativas visando a captar um possível relacionamento linear entre TOT e as variáveis dependentes de valor. Entretanto, os modelos estimados apenas com TOT linear não apresentaram significância para a variável.

A variável INDEP apresentou significância estatística, de $10 \%$, apenas contra a variável de valor Q de Tobin em 1999, sugerindo que as empresas com maior INDEP - proporção de conselheiros externos no conselho apresentaram, em média, maior Q de Tobin em 1999. A constatação da quase inexistência de correlação entre composição do conselho (INDEP) e valor converge com os resultados descritos no estudo de Hermalin e Weisbach (1991, p. 3), que afirmam que um dos principais resultados da literatura empírica sobre conselhos de administração é a ausência de correlação entre composição do conselho e variáveis de desempenho corporativo.
Os resultados apresentados não oferecem evidências conclusivas em favor das hipóteses apresentadas, tendo em vista a insignificância estatística de alguns coeficientes de variáveis de governança em determinados modelos estimados. A causa da ambigüidade das conclusões pode passar pelas limitações metodológicas do estudo, mas também poderia ser atribuída à relativa desimportância conferida pelos agentes dos mercados de capitais a alguns dos elementos da governança corporativa considerados na pesquisa.

Duas variáveis de controle mostraram-se importantes para o aprimoramento da qualidade estatística dos modelos e merecem ser destacadas para possível utilização em estudos posteriores:

- dívida financeira total /ativo total (DIVTSAT): por ser muito correlacionada ao valor e ao desempenho das empresas, mostrou-se importante para todos os modelos construídos;

- setor de serviços de transporte, comunicação e ele-

Tabela 7 - Relação entre a variável valor da empresa sobre ativo total (FIRMVSAT) e as variáveis de governança em 1998.

\begin{tabular}{|l|c|c|c|c|}
\hline \multicolumn{1}{|c|}{ VARIÁVEL } & COEFICIENTE & ERRO PADRÃO & ESTATÍSTICA T & PROB. \\
\hline Constante & 0,228448 & 0,166342 & 1,373361 & 0,1718 \\
\hline DE & $-0,042147$ & 0,024553 & $-1,716569$ & $\mathbf{0 , 0 8 8 2}$ \\
\hline INDEP & $-0,065909$ & 0,049995 & $-1,318314$ & 0,1895 \\
\hline LOG (TOT) & $-0,058554$ & 0,190803 & $-0,306883$ & 0,7594 \\
\hline LOG (TOT)^2 & 0,020037 & 0,053332 & 0,375700 & 0,7077 \\
\hline SERV & 0,177545 & 0,021818 & 8,137488 & 0,0000 \\
\hline EBITSAT2 & 0,409029 & 0,118289 & 3,457885 & 0,0007 \\
\hline DIVTSAT & 0,006926 & 0,000544 & 12,72523 & 0,0000 \\
\hline R-quadrado & 0,606759 & Critério de Schwarz & $-1,313128$ \\
\hline R-quadrado ajustado & 0,587373 & Prob. (estatística F) & 0,000000 \\
\hline
\end{tabular}

Tabela 8 - Testes de heterocedasticidade de White e de normalidade de Jarque-Bera.

\begin{tabular}{|c|c|c|c|c|c|}
\hline ANO & EQUAÇÃO & ESTATÍSTICA WHITE & PROB. (WHITE) & ESTATÍSTICA JARQUE-BERA & PROB. (JARQUE-BERA) \\
\hline \multirow{2}{*}{$\mathbf{2 0 0 0}$} & Tabela 2 & 23,12244 & 0,512580 & 3,094998 & 0,212779 \\
\hline & Tabela 3 & 25,86655 & 0,102849 & 2,146018 & 0,341978 \\
\hline \multirow{2}{*}{1999} & Tabela 4 & 15,21723 & 0,914241 & 4,118014 & 0,127581 \\
\cline { 2 - 6 } & Tabela 5 & 23,60712 & 0,484237 & 2,751403 & 0,252662 \\
\hline \multirow{2}{*}{1998} & Tabela 6 & 28,36794 & 0,244827 & 3,959851 & 0,138080 \\
\hline & Tabela 7 & 36,96086 & 0,250437 & 4,210329 & 0,121826 \\
\hline
\end{tabular}


tricidade (SERV): dentre todas as variáveis binárias setoriais utilizadas para controle, apenas a referente ao setor de serviços apresentou, eventualmente, relação significativa contra as variáveis dependentes.
Novos estudos devem ser feitos na busca da relação entre governança e valor das empresas, utilizando um horizonte de tempo maior ou distinto do utilizado nesta pesquisa. Ademais, procedimentos estatísticos alterna-

Anexo A - Códigos das melhores práticas utilizadas na pesquisa.

\begin{tabular}{|c|c|c|c|c|}
\hline PAÍs & TÍTULO DO CÓDIGO & INSTITUIÇÃO & ANO & DISPONIVEL EM \\
\hline Brasil & $\begin{array}{l}\text { Código das Melhores Práticas de } \\
\text { Governança Corporativa }\end{array}$ & IBGC & 2002 & www.ibgc.org.br \\
\hline Brasil & Cartilha de Governança Corporativa & CVM & 2002 & www.cvm.gov.br \\
\hline EUA & $\begin{array}{l}\text { Corporate Governance } \\
\text { Core Principles \& Guidelines }\end{array}$ & CALPERS & 1998 & $\begin{array}{l}\text { www.calpers-governance.org/ } \\
\text { principles/domestic }\end{array}$ \\
\hline EUA & $\begin{array}{c}\text { Global Corporate } \\
\text { Governance Principles }\end{array}$ & CALPERS & 1999 & $\begin{array}{l}\text { www.calpers-governance.org/ } \\
\text { principles/international/ }\end{array}$ \\
\hline EUA & $\begin{array}{l}\text { TIAA-CREF Policy Statement on } \\
\text { Corporate Governance }\end{array}$ & TIAA-CREF & 2000 & $\begin{array}{l}\text { www.ecgi.de/codes/ } \\
\text { menu_americas.htm }\end{array}$ \\
\hline EUA & Corporate Governance Policies & $\begin{array}{l}\text { Council of Institutional } \\
\text { Investors (CII) }\end{array}$ & 2001 & $\begin{array}{l}\text { www.ecgi.de/codes/ } \\
\text { menu_americas.htm }\end{array}$ \\
\hline EUA & $\begin{array}{l}\text { Statement on Corporate } \\
\text { Governance }\end{array}$ & The Business Roundtable & 1997 & $\begin{array}{l}\text { www.ecgi.de/codes/ } \\
\text { menu_americas.htm }\end{array}$ \\
\hline Reino Unido & $\begin{array}{l}\text { Report of the Committee on the } \\
\text { Financial Aspects of Corporate } \\
\text { Governance - Cadbury Report }\end{array}$ & London Stock Exchange & 1992 & $\begin{array}{l}\text { www.ecgi.de/codes/ } \\
\text { menu_europe.htm }\end{array}$ \\
\hline Reino Unido & Greenbury Recommendations & London Stock Exchange & 1995 & $\begin{array}{l}\text { www.ecgi.de/codes/ } \\
\text { menu_europe.htm }\end{array}$ \\
\hline Reino Unido & $\begin{array}{c}\text { Committee on Corporate Governance - } \\
\text { Hampel Report }\end{array}$ & London Stock Exchange & 1998 & $\begin{array}{l}\text { www.ecgi.de/codes/ } \\
\text { menu_europe.htm }\end{array}$ \\
\hline Reino Unido & $\begin{array}{l}\text { The Combined Code - Principles of Good } \\
\text { Governance and Code of Best Practice }\end{array}$ & London Stock Exchange & 2000 & $\begin{array}{l}\text { www.ecgi.de/codes/ } \\
\text { menu_europe.htm }\end{array}$ \\
\hline União Européia & $\begin{array}{l}\text { Euroshareholders Corporate } \\
\text { Governance Guidelines }\end{array}$ & Euroshareholders & 2000 & $\begin{array}{l}\text { www.ecgi.de/codes/ } \\
\text { menu_europe.htm }\end{array}$ \\
\hline OECD & $\begin{array}{l}\text { OECD Principles of Corporate } \\
\text { Governance }\end{array}$ & OECD & 1999 & www.oecd.org \\
\hline Alemanha & $\begin{array}{l}\text { Code of Best Practice for German } \\
\text { Corporate Governance }\end{array}$ & $\begin{array}{l}\text { German Panel on } \\
\text { Corporate Governance }\end{array}$ & 2000 & $\begin{array}{l}\text { www.ecgi.de/codes/ } \\
\text { menu_europe.htm }\end{array}$ \\
\hline Alemanha & German Code of Corporate Governance & Berlin initiative Group & 2000 & $\begin{array}{l}\text { www.ecgi.de/codes/ } \\
\text { menu_europe.htm }\end{array}$ \\
\hline França & $\begin{array}{l}\text { Recommendations of the Committee on } \\
\text { Corporate Governance - Vienot Report }\end{array}$ & $\begin{array}{l}\text { Association Francaise Des } \\
\text { Entreprises Privers - AFEP }\end{array}$ & 1999 & $\begin{array}{l}\text { www.ecgi.de/codes/ } \\
\text { menu_europe.htm }\end{array}$ \\
\hline Japão & $\begin{array}{l}\text { Corporate Governance Principles - A } \\
\text { Japanese View (Interim Report) }\end{array}$ & $\begin{array}{l}\text { Corporate Governance } \\
\text { Forum of Japan }\end{array}$ & 1997 & $\begin{array}{l}\text { www.ecgi.org/codes/ } \\
\text { menu_asia_pacific.htm }\end{array}$ \\
\hline
\end{tabular}


tivos podem ser aplicados, bem como outras definições operacionais para as variáveis. Como exemplos de alternativas metodológicas, poderia ser aplicado um modelo de equações simultâneas ou modelos para dados em painel. Dada a amplitude da linha de pesquisa, outros estudos podem ser desenvolvidos, procurando responder questões do tipo:

- como avaliar de forma mais precisa a independência do Conselho de Administração em relação aos executivos e /ou acionistas controladores? Na presente pesquisa, dada a limitação da informação pública disponível, a independência do conselho foi aproximada pela proporção de membros externos no conselho, quando melhor seria mensurá-la pela proporção de conselheiros profissionais, uma vez que estes possuem maior probabilidade de terem atitudes independentes;

- como a composição do conselho afeta a execução de suas tarefas (remuneração dos executivos, demissão do diretor executivo, aquisição de outras empresas etc.);

- quais fatores afetam a composição do conselho e como eles evoluem ao longo do tempo? Nesse caso, poderiam ser estudados os impactos de eventos, como mudança na estrutura de propriedade, variação significativa do desempenho da empresa e substituição do diretor executivo na composição do Conselho de Administração das companhias;

- como se pode lidar com a possível relação endógena entre Conselho de Administração e desempenho, haja vista o dinamismo da relação de causalidade entre as variáveis? Assim como a governança corporativa pode ser causa do desempenho corporativo, ela também pode ser conseqüência do mesmo, uma vez que as empresas podem alterar sua estrutura de governança em função de variações de seu desempenho. O não tratamento do problema suspeito da endogeneidade entre as variáveis constitui-se em uma limitação do presente estudo. Uma forma promissora de lidar com a questão envolve a realização de testes com período de tempo defasados, buscando relacionar a estrutura de governança das companhias em um determinado instante com o desempenho corporativo em um instante anterior ou subseqüente; e

- que outros fatores - estágio de vida da empresa, posicionamento competitivo, setor de atuação - podem influenciar a estrutura de governança das companhias? As empresas desenvolveriam estruturas de governança ótimas para suas circunstâncias?

\section{Artigo recebido em 14.11.2002. Aprovado em 14.04.2003.}

\section{Notas}

1. O presente artigo aborda a governança corporativa sob uma estrita perspectiva de agência. Para uma discussão teórica mais profunda, ver Jensen (2001), La Porta et al. (2000) e Shleifer e Vishny (1997).

2. Para uma discussão teórica e metodológica sobre o Q de Tobin, ver Famá e Barros (2000).

3. Embora pareça excessivamente pequeno, o valor de $0,001 \%$ como ponto de corte reflete a grande concentração dos negócios da Bovespa em alguns poucos papéis. De fato, um número elevado de empresas listadas apresentou nível de liquidez bastante inferior a esse mínimo, resultando em sua exclusão na população relevante para a pesquisa.

4. Em junho de 2002 havia 410 empresas listadas na Bolsa de Valores de São Paulo.

5. A adequação dos modelos ao pressuposto de normalidade dos termos de erro em todos os anos da pesquisa só pôde ser obtida mediante a exclusão de algumas observações extremas. Não obstante, os resultados com ou sem outliers são qualitativamente similares, ou seja, sugerem as mesmas conclusões gerais.

\section{Referências Bibliográficas}

BARNHART, Scott e ROSENSTEIN, Stuart. Board composition, managerial ownership and firm performance: an empirical analysis. The Financial Review, n. 33, p. 1-16, Nov. 1998.

BAYSINGER, B. e BUTLER, H. Corporate governance and the board of directors: performance effects of changes in board composition. Journal of Law, Economics and Organization, v. 1, p. 101-24, 1985.

BHAGAT, Sanjai e BLACK, Bernard. The uncertain relationship between board composition and firm performance. Business Lawyer, v. 54, p. 921-63, 1999.

BHAGAT, Sanjai e BLACK, Bernard. Board independence and long term firm performance. Journal of Corporation Law (Forthcoming), v. 27, n. 1, 2002.

BROWN, William e MALONEY, Michael. Exit, voice, and the role of corporate directors: evidence from acquisition performance, 1998. Unpublished working paper.

CHUNG, Kee e PRUITT, Stephen. A simple approximation of Tobin's Q. Financial Management, v. 23, n. 3, p. 70-4, Autumn 1994. 
DALTON, Dan e DAILY, Catherine. The board and financial performance: bigger is better. NACD Director's Monthly, p. 1-5, Aug. 2000.

EISENBERG, Theodore, SUNDGREN, Stefan e WELLS, Martin. Larger board size and decreasing firm value in small firms. Journal of Financial Economics, v. 48, p. 35-54, 1998.

FAMÁ, Rubens e BARROS, Lucas Ayres. Q de Tobin e seu uso em finanças: aspectos metodológicos e conceituais. Cadernos de Pesquisa em Administração, v. 7, n. 4, p. 27-43, Out. /Dez. 2000.

FÜERST, Oren e KANG, Sok-Hyon. Corporate governance, expected operating performance, and pricing. Work in Progress, Yale School of Management, New Haven (CT), v. 2, n. 2, 2000.

HERMALIN, Benjamin e WEISBACH, Michael. The effect of board composition and direct incentives on firm performance. Financial Management, v. 21, n. 4, p. 101-12, 1991.

JENSEN, Michael e MECKLING, William. Theory of the firm: managerial behavior, agency costs and ownership structure. Journal of Financial Economics, v. 3, p. 305-60, Oct. 1976.
JENSEN, Michael. The modern industrial revolution, exit, and the failure of internal control systems. Journal of Finance, v. 48, p. 83180, 1993.

JENSEN, Michael. A theory of the firm: governance, residual claims, and organizational forms. 1st. ed. Boston : Harvard University Press, 2001.320p

LA PORTA, Rafael, SHLEIFER, Andrei, LOPEZ-DE-SILANES, Florencio e VISHNY, Robert. Investor protection and corporate governance. Journal of Financial Economics, v. 58, p. 3-27, Oct. 2000.

SHLEIFER, Andrei e VISHNY, Robert. A survey of corporate governance. Journal of Finance, v. 52, n. 2, p. 737-83, 1997.

TOBIN, James. A general equilibrium approach to monetary theory. Journal of Money, Credit, and Banking, v. 1, n. 1, p. 15-29, Feb. 1969.

TOBIN, James e BRAINARD, W. Pitfalls in financial model building, American Economic Review, v. 58, n. 2, p. 99-122, May 1968.

YERMACK, David. Higher market valuation of companies with a small board of Directors. Journal of Financial Economics, v. 40, n. 3 , p. 185-213, 1996.

\author{
Alexandre Di Miceli da Silveira \\ Doutorando e Mestre em Administração de Empresas FEA/USP. Interesses de Pesquisa: Governança Corporativa, Finanças \\ Corporativas e Avaliação de Empresas. \\ E-mail: alexfea@usp.br \\ Endereço: R. Afonso de Freitas, 320, ap. 71. São Paulo, SP. CEP 04006-051. \\ Lucas Ayres B. de C. Barros \\ Doutorando e Mestre em Administração de Empresas FEA/USP. Interesses de Pesquisa: Finanças Econômicas e Corporativas. \\ E-mail: lucasayres@hotmail.com \\ Endereço: R. Florália, 103. São Paulo, SP. CEP 05451-130.

\section{Rubens Famá} \\ Professor Doutor do Departamento de Administração de Empresas pela FEA/USP nos programas de graduação e pós- \\ graduação. Interesses de Pesquisa: Finanças Econômicas e Corporativas, Governança Corporativa \\ E-mail: rfama@usp.br \\ Endereço: R. Morás, 656, ap. 5. São Paulo, SP. CEP 05434-020.
}

